Mathematical Modelling and Analysis

Volume 22 Number 6, November 2017, 733-749

https://doi.org/10.3846/13926292.2017.1365313

(c) Vilnius Gediminas Technical University, 2017
Publisher: Taylor\&Francis and VGTU

http://www.tandfonline.com/TMMA

ISSN: $1392-6292$

eISSN: $1648-3510$

\title{
On Zeros of Some Combinations of Dirichlet $L$-Functions and Hurwitz Zeta-Functions
}

\section{Virginija Garbaliauskienè $\dot{e}^{a}$, Julija Karaliūnaite $\dot{\mathrm{e}}^{b}$ and Antanas Laurinčikas ${ }^{c}$}

${ }^{a}$ Faculty of Technology, Physical and Biomedical Sciences, Šiauliai University Vilniaus str. 141, LT-76353 Šiauliai, Lithuania

${ }^{b}$ Faculty of Fundamental Sciences, Vilnius Gediminas Technical University

Saulètekio al. 11, LT-10223 Vilnius, Lithuania

${ }^{c}$ Faculty of Mathematics and Informatics, Vilnius University

Naugarduko str. 24, LT-03225 Vilnius, Lithuania

E-mail(corresp.): vgarbaliauskiene@gmail.com

E-mail: julija.karaliunaite@vgtu.lt

Received June 4, 2017; revised August 3, 2017; published online November 15, 2017

Abstract. In the paper, we obtain that certain linear and more general combinations of Dirichlet $L$-functions and Hurwitz zeta-functions have infinitely many zeros in the critical strip. For the proof, universality of those combinations is applied.

Keywords: Dirichlet $L$-function, Hurwitz zeta-function, Mergelyan theorem, Rouché theorem, universality.

AMS Subject Classification: 11M06; 11M85.

\section{Introduction}

Let $s=\sigma+i t$ be a complex variable, $\chi$ be a Dirichlet character and $\alpha, 0<$ $\alpha \leq 1$, be a fixed parameter. The Dirichlet $L$-function $L(s, \chi)$ and Hurwitz zeta-function $\zeta(s, \alpha)$ are defined, for $\sigma>1$, by the series

$$
L(s, \chi)=\sum_{m=1}^{\infty} \frac{\chi(m)}{m^{s}} \quad \text { and } \quad \zeta(s, \alpha)=\sum_{m=0}^{\infty} \frac{1}{(m+\alpha)^{s}}
$$

and continue meromorphically to the whole complex plane. If $\chi(m)$ is a nonprincipal character, then $L(s, \chi)$ is an entire function, while if $\chi(m)$ is the principal character modulo $q$, then $L(s, \chi)$ has a simple pole at the point $s=1$ with residue $\prod_{p \mid q}(1-1 / p)$, where $p$ denotes a prime number. The function $\zeta(s, \alpha)$ has a simple pole at the point $s=1$ with residue 1 .

It is well known that $L(s, \chi) \neq 0$ in the half-plane $\sigma>1$. Moreover, the function $L(s, \chi)$ has infinitely many zeros lying in the critical strip $\{s \in \mathbb{C}$ : 
$0<\sigma<1\}$. The generalized Riemann hypothesis asserts that all these zeros lie on the critical line.

The properties of the function $\zeta(s, \alpha)$, including the zero-distribution, depend on the arithmetical nature of the parameter $\alpha$. In [5], it was proved that the function $\zeta(s, \alpha)$ with transcendental or rational parameter $\alpha \neq 1, \frac{1}{2}$ has infinitely many zeros in the half-plane $\{s \in \mathbb{C}: \sigma>1\}$. J.W.S. Cassels extended [4] the latter result for the case of algebraic irrational $\alpha$. The above results also can be found in [14].

It is also known that the function $\zeta(s, \alpha)$ has zeros in the strip $D=$ $\left\{s \in \mathbb{C}: \frac{1}{2}<\sigma<1\right\}$. Let $\alpha=\frac{a}{q},(a, q)=1$ and $0<a<q$. Then S.M. Voronin in his thesis [22], see also [23], obtained, that, for every $\sigma_{1}, \sigma_{2}, \frac{1}{2}<\sigma_{1}<\sigma_{2}<1$, there exists a constant $c=c\left(\alpha, \sigma_{1}, \sigma_{2}\right)>0$ such that, for sufficiently large $T$, the function $\zeta(s, \alpha)$ has more than $c T$ zeros lying in the rectangle $\{s \in \mathbb{C}$ : $\left.\sigma_{1}<\sigma<\sigma_{2},|t|<T\right\}$. S.M. Gonek in [6] proved an analogical result in the case of transcendental $\alpha$.

The case of algebraic irrational $\alpha$ remains an open problem. The same results on zeros of $\zeta(s, \alpha)$ in the strip $D$ were obtained independently by B. Bagchi in his thesis [1], they also can be found in [14]. Proofs of the statements on zero distribution of $\zeta(s, \alpha)$ in $D$ are based on the universality property of $\zeta(s, \alpha)$. Denote by meas $A$ the Lebesgue measure of a measurable set $A \subset \mathbb{R}$. Then the universality of $\zeta(s, \alpha)$ is contained in the following statement. Suppose that $\alpha$ is rational $\neq 1, \frac{1}{2}$ or transcendental number. Let $K \subset D$ be a compact subset with connected complement, and let $f(s)$ be a continuous function on $K$ which is analytic in the interior of $K$. Then, for every $\varepsilon>0$,

$$
\liminf _{T \rightarrow \infty} \frac{1}{T} \operatorname{meas}\left\{\tau \in[0, T]: \sup _{s \in K}|\zeta(s+i \tau, \alpha)-f(s)|<\varepsilon\right\}>0 .
$$

The later theorem for rational $\alpha$ in non-explicit form was proved by S.M. Voronin [22]. Both the cases of rational and transcendental $\alpha$ by different methods were treated in [6] and [1].

Clearly, $\zeta(s, 1)=\zeta(s)$ and $\zeta\left(s, \frac{1}{2}\right)=\left(2^{s}-1\right) \zeta(s)$, where $\zeta(s)$ is the Riemann zeta-function. Therefore, in those cases, $\zeta(s, \alpha)$ is also universal, however, the approximated function $f(s)$ must be non-vanishing on $K$. From this, it follows that the universality can not be used to detect zeros of $\zeta(s, \alpha)$ in $D$ if $\alpha=1$ or $\alpha=\frac{1}{2}$.

The present paper is devoted to zeros of certain combinations and more general functions of Dirichlet $L$-functions and Hurwitz zeta-functions.

We say that, for a function $L(s)$, the assertion $A\left(\sigma_{1}, \sigma_{2} ; c, T\right)$ is valid if, for every $\sigma_{1}, \sigma_{2}, \frac{1}{2}<\sigma_{1}<\sigma_{2}<1$, there exists a constant $c>0$ such that, for sufficiently large $T$, the function $L(s)$ has more than $c T$ zeros lying in the rectangle $\left\{s \in \mathbb{C}: \sigma_{1}<\sigma<\sigma_{2}, 0<t<T\right\}$.

A lot of results on the number of zeros of linear combinations of universal functions can be found in [18]. The paper [19] also is rich by interesting results on zeros of some polynomials of universal functions. For example, a partial case of a corollary of Theorem 2 from [18] is the following theorem. 
Theorem 1. Suppose that $\chi_{1}, \ldots, \chi_{n}$ are pairwise non-equivalent Dirichlet characters, and $P_{1}(s), \ldots, P_{n}(s)$ are not identically vanishing general Dirichlet series which are absolutely convergent for $\sigma>\frac{1}{2}$, and at least two of the series $P_{j}(s)$ are non-vanishing in the strip $D$. Then there exists a constant $c=c\left(\chi_{1}, \ldots, \chi_{n}, P_{1}, \ldots, P_{n}, \sigma_{1}, \sigma_{2}\right)>0$ such that, for the linear combination

$$
P_{1}(s) L\left(s, \chi_{1}\right)+\cdots+P_{n}(s) L\left(s, \chi_{n}\right)
$$

the assertion $A\left(\sigma_{1}, \sigma_{2} ; c, T\right)$ is valid.

Denote by $H(G)$ the space of analytic functions on $G$ endowed with the topology of uniform convergence on compacta. Let

$$
S=\left\{g \in H(D): \frac{1}{g(s)} \in H(D) \text { or } g(s) \equiv 0\right\}
$$

and denote by $U_{n}$ the class of continuous operators $F: H^{n}(D) \rightarrow H(D)$ such that, for every open set $G \subset H(D)$,

$$
\left(F^{-1} G\right) \cap S^{n} \neq \varnothing .
$$

Theorem 2. Suppose that $\chi_{1}, \ldots, \chi_{n}$ are pairwise non-equivalent Dirichlet characters, and that $F \in U_{n}$. Then there exists a constant $c=c\left(\chi_{1}, \ldots, \chi_{n}, F\right.$, $\left.\sigma_{1}, \sigma_{2}\right)>0$ such that, for the function $F\left(L\left(s, \chi_{1}\right), \ldots, L\left(s, \chi_{n}\right)\right)$, the assertion $A\left(\sigma_{1}, \sigma_{2} ; c, T\right)$ is valid.

The class $U_{n}$ is theoretical, it is difficult to check its hypotheses. Now we define a simpler class of operators $F$. Let $V$ be an arbitrary positive number, $D_{V}=\left\{s \in \mathbb{C}: \frac{1}{2}<\sigma<1,|t|<V\right\}$ and

$$
S_{V}=\left\{g \in H\left(D_{V}\right): \frac{1}{g(s)} \in H\left(D_{V}\right) \text { or } g(s) \equiv 0\right\} .
$$

We say that the continuous operator $F: H^{n}\left(D_{V}\right) \rightarrow H\left(D_{V}\right)$ belongs to the class $U_{n, V}$ if, for every polynomial $p=p(s)$,

$$
\left(F^{-1}\{p\}\right) \cap S_{V}^{n} \neq \varnothing .
$$

Theorem 3. Suppose that $\chi_{1}, \ldots, \chi_{n}$ are pairwise non-equivalent Dirichlet characters, and that $F \in U_{n, V}$ with sufficiently large $V$. Then there exists a constant $c=c\left(\chi_{1}, \ldots, \chi_{n}, F, \sigma_{1}, \sigma_{2}\right)>0$ such that, for the function $F\left(L\left(s, \chi_{1}\right)\right.$, $\left.\ldots, L\left(s, \chi_{n}\right)\right)$ the assertion $A\left(\sigma_{1}, \sigma_{2} ; c, T\right)$ is valid with $T<V$.

We give an example of $F \in U_{V}$. Let $F\left(g_{1}, g_{2}\right)=g_{1}^{2}+g_{2}^{2}, g_{1}, g_{2} \in H\left(D_{V}\right)$. Let $p(s)$ be an arbitrary polynomial. Then there exists a constant $c_{1}>0$ such that $|p(s)| \leq c_{1}$ for $s \in D_{V}$. We take $C>c_{1}$, and

$$
p_{1}(s)=\frac{p(s)+C}{2 \sqrt{C}}, \quad p_{2}(s)=\frac{p(s)-C}{2 i \sqrt{C}} .
$$

Then we have that $p_{1}(s) \neq 0$ and $p_{2}(s) \neq 0$ on $D_{V}$. Moreover,

$$
p_{1}^{2}(s)+p_{2}^{2}(s)=p(s) .
$$


Thus, $\left(p_{1}, p_{2}\right) \in\left(F^{-1}\{p\}\right) \cap S_{V}^{2}$. Therefore, if $\chi_{1}$ and $\chi_{2}$ are non-equivalent characters, then the function

$$
L^{2}\left(s, \chi_{1}\right)+L^{2}\left(s, \chi_{2}\right)
$$

has more than $c T$ zeros ir the rectangle $\left\{s \in \mathbb{C}: \sigma_{1}<\sigma<\sigma_{2}, 0<t<T\right\}$ with sufficiently large $T<V$.

The second example is of the following form. Let $P_{1}(s), \ldots, P_{n}(s)$ be analytic functions on $D$, two of them are non-vanishing, and the remaining functions are bounded by polynomials. Then, for the function

$$
P_{1}(s) L\left(s, \chi_{1}\right)+\cdots+P_{n}(s) L\left(s, \chi_{n}\right),
$$

where $\chi_{1}, \ldots, \chi_{n}$ are pairwise non-equivalent Dirichlet characters, the assertion $A\left(\sigma_{1}, \sigma_{2} ; c, T\right)$ is valid. Indeed, the operator $F: H^{n}\left(D_{V}\right) \rightarrow H\left(D_{V}\right)$ given by the formula

$$
F\left(g_{1}, \ldots, g_{n}\right)=P_{1} g_{1}+\cdots+P_{n} g_{n}, \quad g_{1}, \ldots, g_{n} \in H\left(D_{V}\right),
$$

is continuous. Let $p=p(s)$ be an arbitrary polynomial. Without loss of generality, we can suppose that $P_{1}(s)$ and $P_{2}(s)$ are non-vanishing for $s \in D$. Moreover, there exist polynomials $q_{j}(s), j=3, \ldots, n$, such that, for $s \in D$, $\left|P_{j}(s)\right| \leq\left|q_{j}(s)\right|, j=3, \ldots, n$. We take

$$
\begin{aligned}
& g_{1}(s)=\frac{p(s)+C}{P_{1}(s)}, \quad g_{2}(s)=\frac{-\left(P_{3}(s)+\cdots+P_{n}(s)\right)-C}{P_{2}(s)}, \\
& g_{3}(s)=\cdots=g_{n}(s)=1,
\end{aligned}
$$

where $C>0$ is such that $p(s)+C \neq 0$ and $-\left(P_{3}(s)+\cdots+P_{n}(s)\right)-C \neq 0$ on $D_{V}$. Then we have that

$$
P_{1} g_{1}+\cdots+P_{n} g_{n}=p
$$

and $g_{1}, \ldots, g_{n} \neq 0$ on $D_{V}$. Therefore the hypotheses of Theorem 3 are satisfied.

Now we state the results on zeros of certain functions related to Hurwitz zeta-functions. Define

$$
L\left(\alpha_{1}, \ldots, \alpha_{n}\right)=\left\{\log \left(m+\alpha_{j}\right): m \in \mathbb{N}_{0}=\mathbb{N} \cup\{0\}, j=1, \ldots, n\right\} .
$$

Theorem 4. Suppose that the set $L\left(\alpha_{1}, \ldots, \alpha_{n}\right)$ is linearly independent over the field of rational numbers $\mathbb{Q}$, and $c_{1}, \ldots, c_{n}$ are complex numbers, at least one of them is non-zero. Then there exists a constant $c=c\left(\alpha_{1}, \ldots, \alpha_{n}, c_{1}, \ldots\right.$, $\left.c_{n}, \sigma_{1}, \sigma_{2}\right)>0$ such that, for the function

$$
c_{1} \zeta\left(s, \alpha_{1}\right)+\cdots+c_{n} \zeta\left(s, \alpha_{n}\right)
$$

the assertion $A\left(\sigma_{1}, \sigma_{2} ; c, T\right)$ is valid.

Denote by $\hat{U}_{n}$ the class of continuous operators $F: H^{n}(D) \rightarrow H(D)$ such that, for every open set $G \subset H(D)$, the set $F^{-1} G$ is non-empty. 
Theorem 5. Suppose that the set $L\left(\alpha_{1}, \ldots, \alpha_{n}\right)$ is linearly independent over $\mathbb{Q}$, and that $F \in \hat{U}_{n}$. Then there exists a constant $c=c\left(\alpha_{1}, \ldots, \alpha_{n}, F, \sigma_{1}, \sigma_{2}\right)>$ 0 such that, for the function $F\left(\zeta\left(s, \alpha_{1}\right), \ldots, \zeta\left(s, \alpha_{n}\right)\right)$, the assertion $A\left(\sigma_{1}, \sigma_{2}\right.$; $c, T)$ is valid.

Now we consider a simpler class of operators. Denote by $\hat{U}_{n 1}$ the class of continuous operators $F: H^{n}(D) \rightarrow H(D)$ such that each polynomial $p(s)$ has its preimage $F^{-1}\{p\}$.

Theorem 6. Suppose that the set $L\left(\alpha_{1}, \ldots, \alpha_{n}\right)$ is linearly independent over $\mathbb{Q}$, and that $F \in \hat{U}_{n 1}$. Then there exists a constant $c=c\left(\alpha_{1}, \ldots, \alpha_{n}, F, \sigma_{1}, \sigma_{2}\right)$ $>0$ such that, for the function $F\left(\zeta\left(s, \alpha_{1}\right), \ldots, \zeta\left(s, \alpha_{n}\right)\right)$, the assertion $A\left(\sigma_{1}, \sigma_{2}\right.$; $c, T)$ is valid.

For example, the operator $F: H^{2}(D) \rightarrow H(D)$ defined by the formula $F\left(g_{1}, g_{2}\right)=g_{1}^{2}+g_{2}^{2}$ belongs to the class $\hat{U}_{21}$. Really, for any polynomial $p(s)$, there exist two polynomials

$$
p_{1}(s)=\frac{p(s)+1}{2} \text { and } p_{2}(s)=\frac{p(s)-1}{2 i},
$$

and $p_{1}^{2}(s)+p_{2}^{2}(s)=p(s)$.

Remark 1. In view of Theorem 6 , the numbers $c_{1}, \ldots, c_{n}$ in Theorem 4 can be replaced by functions analytic in $D$.

Now we consider a collection of functions $L\left(s, \chi_{1}\right), \ldots, L\left(s, \chi_{l}\right), \zeta\left(s, \alpha_{1}\right)$, $\ldots, \zeta\left(s, \alpha_{r}\right)$. Denote by $\mathcal{P}$ the set of all prime numbers, and define the set

$$
L\left(\mathcal{P}, \alpha_{1}, \ldots, \alpha_{r}\right)=\left\{(\log p: p \in \mathcal{P}),\left(\log \left(m+\alpha_{j}\right): m \in \mathbb{N}_{0}, j=1, \ldots, r\right)\right\} .
$$

Theorem 7. Suppose that $\chi_{1}, \ldots, \chi_{l}$ are pairwise non-equivalent Dirichlet characters, and that the set $L\left(\mathcal{P}, \alpha_{1}, \ldots, \alpha_{r}\right)$ is linearly independent over $\mathbb{Q}$. Let $c_{1}, \ldots, c_{l}, \hat{c}_{1}, \ldots, \hat{c}_{r}$ be complex numbers, at least one of $\hat{c}_{j}$ is non-zero. Then there exists a positive constant $c=c\left(\chi_{1}, \ldots, \chi_{l}, \alpha_{1}, \ldots, \alpha_{r}, c_{1}, \ldots, c_{l}, \hat{c}_{1}, \ldots, \hat{c}_{r}\right.$, $\left.\sigma_{1}, \sigma_{2}\right)>0$ such that, for the function

$$
c_{1} L\left(s, \chi_{1}\right)+\cdots+c_{l} L\left(s, \chi_{l}\right)+\hat{c}_{1} \zeta\left(s, \alpha_{1}\right)+\cdots+\hat{c}_{r} \zeta\left(s, \alpha_{r}\right)
$$

the assertion $A\left(\sigma_{1}, \sigma_{2} ; c, T\right)$ is valid.

We say that a continuous operator $F: H^{l+r}(D) \rightarrow H(D)$ belongs to the class $U_{l, r}$ if, for every polynomial $p(s)$, the set $\left(F^{-1}\{p\}\right) \cap\left(S^{l} \times H^{r}(D)\right)$ is non-empty.

Theorem 8. Suppose that $\chi_{1}, \ldots, \chi_{l}$ are pairwise non-equivalent Dirichlet characters, the set $L\left(\mathcal{P}, \alpha_{1}, \ldots, \alpha_{r}\right)$ is linearly independent over $\mathbb{Q}$, and that $F \in$ $U_{l, r}$. Then there exists a constant $c=c\left(\chi_{1}, \ldots, \chi_{l}, \alpha_{1}, \ldots, \alpha_{r}, F, \sigma_{1}, \sigma_{2}\right)>0$ such that, for the function

$$
F\left(L\left(s, \chi_{1}\right), \ldots L\left(s, \chi_{l}\right), \zeta\left(s, \alpha_{1}\right) \ldots, \zeta\left(s, \alpha_{r}\right)\right)
$$

the assertion $A\left(\sigma_{1}, \sigma_{2} ; c, T\right)$ is valid. 
For example, if $\chi_{1}$ and $\chi_{2}$ are non-equivalent characters, and the numbers $\alpha_{1}$ and $\alpha_{2}$ are algebraically independent over $\mathbb{Q}$, then the function $L\left(s, \chi_{1}\right)$ $L\left(s, \chi_{2}\right) \zeta\left(s, \alpha_{1}\right) \zeta\left(s, \alpha_{2}\right)$ satisfies the hypotheses of Theorem 8, since a polynomial $p(s)$ has a preimage $(1,1,1, p(s)) \in S^{2} \times H^{2}(D)$, and the algebraic independence of the numbers $\alpha_{1}$ and $\alpha_{2}$ implies the linear independence of the set $L\left(\mathcal{P}, \alpha_{1}, \alpha_{2}\right)$.

Remark 2. In view of Theorem 8, the numbers $c_{j}$ and $\hat{c}_{j}$ in Theorem 7 can be replaced by functions analytic in $D$.

\section{Universality}

In Introduction, we have mentioned the universality of the Hurwitz zeta-function. In this section, we present more general universality results which will be applied for the proof of Theorems 1-8. We start with a joint universality theorem for Dirichlet $L$-functions. In this theorem, a collection of shifts of Dirichlet $L$-functions with non-equivalent Dirichlet characters, uniformly on compact sets of the strip $D$, can approximate simultaneously a collection of non-vanishing analytic functions.

Theorem 9. Suppose that $\chi_{1}, \ldots, \chi_{n}$ are pairwise non-equivalent Dirichlet characters. For $j=1, \ldots, n$, let $K_{j}$ be a compact set of the strip $D$ with connected complement, and let $f_{j}(s)$ be a continuous non-vanishing function on $K_{j}$ which is analytic in the interior of $K_{j}$. Then, for every $\varepsilon>0$,

$$
\liminf _{T \rightarrow \infty} \frac{1}{T} \operatorname{meas}\left\{\tau \in[0, T]: \sup _{1 \leq j \leq n} \sup _{s \in K_{j}}\left|L\left(s+i \tau, \chi_{j}\right)-f_{j}(s)\right|<\varepsilon\right\}>0 .
$$

Theorem 9 in a weaker non-explicit form has been obtained by S.M. Voronin in [21], and applied for the functional independence of Dirichlet $L$-functions. First in a weaker explicit form the theorem was given in [22], see also [23]. Similar results independently by different methods were obtained by S.M. Gonek [6] and B. Bagchi $[1,2]$. In the present form, the theorem was stated in [20] and discussed in [11]. A full proof of Theorem 9 is given in the master work [17].

Theorem 10. Suppose that $\chi_{1}, \ldots, \chi_{n}$ are pairwise non-equivalent Dirichlet characters, and that $F \in U_{n}$. Let $K \subset D$ be a compact set with connected complement, and let $f(s)$ be a continuous function on $K$ which is analytic in the interior of $K$. Then, for every $\varepsilon>0$,

$$
\begin{array}{r}
\liminf _{T \rightarrow \infty} \frac{1}{T} \operatorname{meas}\left\{\tau \in[0, T]: \sup _{s \in K} \mid F\left(L\left(s+i \tau, \chi_{1}\right), \ldots, L\left(s+i \tau, \chi_{n}\right)\right)\right. \\
-f(s) \mid<\varepsilon\}>0 .
\end{array}
$$

The theorem is proved in [11], Theorem 4 .

Theorem 11. Suppose that $\chi_{1}, \ldots, \chi_{n}$ are pairwise non-equivalent Dirichlet characters, and that $K$ and $f(s)$ are the same as in Theorem 10. Let $V>0$ be such that $K \subset D_{V}$, and that $F \in U_{n, V}$. Then the same assertion as in Theorem 10 is valid. 
The proof of the theorem is given in [11], Theorem 5 .

Now we state joint universality theorems for Hurwitz zeta-functions.

Theorem 12. Suppose that the set $L\left(\alpha_{1}, \ldots, \alpha_{n}\right)$ is linearly independent over $\mathbb{Q}$. For $j=1, \ldots, n$, let $K_{j}$ be a compact subset of the strip $D$ with connected complement, and let $f_{j}(s)$ be a continuous function on $K_{j}$ which is analytic in the interior of $K_{j}$. Then, for every $\varepsilon>0$,

$$
\liminf _{T \rightarrow \infty} \frac{1}{T} \operatorname{meas}\left\{\tau \in[0, T]: \sup _{1 \leq j \leq n} \sup _{s \in K_{j}}\left|\zeta\left(s+i \tau, \alpha_{j}\right)-f_{j}(s)\right|<\varepsilon\right\}>0 .
$$

The theorem is proved in [9].

Theorem 12 implies the following two universality theorems for composite functions obtained in [12].

Theorem 13. Suppose that the set $L\left(\alpha_{1}, \ldots, \alpha_{n}\right)$ is linearly independent over $\mathbb{Q}$, and that $F \in \hat{U}_{n}$. Let $K$ and $f(s)$ be the same as in Theorem 10. Then, for every $\varepsilon>0$,

$$
\begin{array}{r}
\liminf _{T \rightarrow \infty} \frac{1}{T} \operatorname{meas}\left\{\tau \in[0, T]: \sup _{s \in K} \mid F\left(\zeta\left(s+i \tau, \alpha_{1}\right), \ldots, \zeta\left(s+i \tau, \alpha_{n}\right)\right)\right. \\
-f(s) \mid<\varepsilon\}>0 .
\end{array}
$$

Theorem 14. Suppose that the set $L\left(\alpha_{1}, \ldots, \alpha_{n}\right)$ is linearly independent over $\mathbb{Q}$, and that $F \in \hat{U}_{n 1}$. Let $K$ and $f(s)$ be the same as in Theorem 10 . Then the same assertion as in Theorem 13 is true.

In [12], theorems on universality of $F\left(\zeta\left(s, \alpha_{1}\right), \ldots, \zeta\left(s, \alpha_{n}\right)\right)$ for some other classes of operators $F$ also can be found.

Denote by $\mathcal{B}(S)$ the class of Borel sets of the space $S$, and on $\left(H^{l+r}(D)\right.$, $\left.\mathcal{B}\left(H^{l+r}(D)\right)\right)$ define the probability measure $P_{T}$ by

$$
\begin{aligned}
P_{T}(A)= & \frac{1}{T} \operatorname{meas}\left\{\tau \in[0, T]:\left(L\left(s+i \tau, \chi_{1}\right), \ldots, L\left(s+i \tau, \chi_{l}\right),\right.\right. \\
& \left.\left.\zeta\left(s+i \tau, \alpha_{1}\right), \ldots, \zeta\left(s+i \tau, \alpha_{r}\right)\right) \in A\right\}, \quad A \in \mathcal{B}\left(H^{l+r}(D)\right) .
\end{aligned}
$$

To state a limit theorem for the measure $P_{T}$, we need some notation and definitions. Denote by $\gamma$ the unit circle on the complex plane, and define

$$
\hat{\Omega}=\prod_{p} \gamma_{p} \quad \text { and } \quad \Omega=\prod_{m=0}^{\infty} \gamma_{m}
$$

where $\gamma_{p}=\gamma$ for all primes $p$, and $\gamma_{m}=\gamma$ for all $m \in \mathbb{N}_{0}$. The tori $\hat{\Omega}$ and $\Omega$ with the product topology and pointwise multiplication are compact topological Abelian groups. Let $\underline{\Omega}=\hat{\Omega} \times \Omega_{1} \times \cdots \times \Omega_{r}$, where $\Omega_{j}=\Omega$ for $j=1, \ldots, r$. Then again $\underline{\Omega}$ is a compact topological Abelian group. Therefore, on $(\underline{\Omega}, \mathcal{B}(\underline{\Omega}))$, the probability Haar measure $m_{H}$ can be defined, and this leads to the probability space $\left(\underline{\Omega}, \mathcal{B}(\underline{\Omega}), m_{H}\right)$. Note that the measure $m_{H}$ is the 
product of the Haar measures $\hat{m}_{H}$ and $m_{j H}$ on $(\hat{\Omega}, \mathcal{B}(\hat{\Omega}))$ and $\left(\Omega_{j}, \mathcal{B}\left(\Omega_{j}\right)\right)$, respectively, $j=1, \ldots, r$. Denote elements of $\underline{\Omega}$ by $\underline{\omega}=\left(\hat{\omega}, \omega_{1}, \ldots, \omega_{r}\right)$, where $\hat{\omega} \in \hat{\Omega}$, and $\omega_{j} \in \Omega_{j}, j=1, \ldots, r$. Moreover, let $\hat{\omega}(p)$ and $\omega_{j}(m)$ be the projections of $\hat{\omega} \in \hat{\Omega}$ to $\gamma_{p}$ and $\omega_{j} \in \Omega_{j}$ to $\gamma_{m}$, respectively, $j=1, \ldots, r$. Now, on the probability space $\left(\underline{\Omega}, \mathcal{B}(\underline{\Omega}), m_{H}\right)$, define the $H^{l+r}(D)$ valued random element $\Xi(s, \underline{\omega})$ by the formula

$$
\Xi(s, \underline{\omega})=\left(L\left(s, \hat{\omega}, \chi_{1}\right), \ldots, L\left(s, \hat{\omega}, \chi_{l}\right), \zeta\left(s, \alpha_{1}, \omega_{1}\right), \ldots, \zeta\left(s, \alpha_{r}, \omega_{r}\right)\right),
$$

where

$$
L\left(s, \hat{\omega}, \chi_{j}\right)=\prod_{p}\left(1-\frac{\chi_{j}(p) \hat{\omega}(p)}{p^{s}}\right)^{-1}, \quad j=1, \ldots, l,
$$

and

$$
\zeta\left(s, \alpha_{j}, \omega_{j}\right)=\sum_{m=0}^{\infty} \frac{\omega_{j}(m)}{\left(m+\alpha_{j}\right)^{s}}, \quad j=1, \ldots, r .
$$

Let $P_{\Xi}$ be the distribution of the random element $\Xi(s, \underline{\omega})$, i.e., for $A \in \mathcal{B}\left(H^{l+r}\right.$ $(D))$,

$$
P_{\Xi}(A)=m_{H}(\underline{\omega} \in \underline{\Omega}: \Xi(s, \underline{\omega}) \in A) .
$$

Proposition 1. Suppose that the set $L\left(\mathcal{P}, \alpha_{1}, \ldots, \alpha_{r}\right)$ is linearly independent over $\mathbb{Q}$. Then the measure $P_{T}$ converges weakly to $P_{\Xi}$ as $T \rightarrow \infty$.

Proof of the proposition is based on the following lemmas.

Lemma 1. Suppose that the set $L\left(\mathcal{P}, \alpha_{1}, \ldots, \alpha_{r}\right)$ is linearly independent over $\mathbb{Q}$. Then the probability measure

$$
\begin{gathered}
Q_{T}(A) \stackrel{\text { def }}{=} \frac{1}{T} \operatorname{meas}\left\{\tau \in[0, T]:\left(\left(p^{-i \tau}: p \in \mathcal{P}\right),\left(\left(m+\alpha_{1}\right)^{-i \tau}: m \in \mathbb{N}_{0}\right),\right.\right. \\
\left.\left.\ldots,\left(\left(m+\alpha_{r}\right)^{-i \tau}: m \in \mathbb{N}_{0}\right)\right) \in A\right\}, \quad A \in \mathcal{B}(\underline{\Omega}),
\end{gathered}
$$

converges weakly to the Haar measure $m_{H}$ as $T \rightarrow \infty$.

Proof. As in the proof of Theorem 3 in [10], we have that the Fourier transform $g_{T}\left(\underline{k}, \underline{l}_{1}, \ldots, \underline{l}_{r}\right), \underline{k}=\left(k_{p}: p \in \mathcal{P}\right), \underline{l}_{1}=\left(l_{1 m}: m \in \mathbb{N}_{0}\right), \ldots, \underline{l}_{r}=\left(l_{r m}\right.$ : $m \in \mathbb{N}_{0}$ ) of the measure $Q_{T}$ is given by

$$
\begin{aligned}
g_{T}\left(\underline{k}, \underline{l}_{1}, \ldots, \underline{l}_{r}\right)=\int_{\underline{\Omega}}\left(\prod_{p \in \mathcal{P}} \hat{\omega}^{k_{p}}(p) \prod_{j=1}^{r} \prod_{m=0}^{\infty} \omega_{j}^{l_{j m}}(m)\right) \mathrm{d} Q_{T} \\
=\frac{1}{T} \int_{0}^{T} \exp \left\{-i \tau\left(\sum_{p \in \mathcal{P}} k_{p} \log p+\sum_{j=1}^{r} \sum_{m=0}^{\infty} l_{j m} \log \left(m+\alpha_{j}\right)\right)\right\} \mathrm{d} \tau
\end{aligned}
$$

where only a finite number of integers $k_{p}$ and $l_{j m}, j=1, \ldots, r$, are distinct from zero. Now we use essentially the fact that the set $L\left(\mathcal{P}, \alpha_{1}, \ldots, \alpha_{r}\right)$ is linearly independent over $\mathbb{Q}$, and, after integration, we obtain from (2.1) that

$$
\lim _{T \rightarrow \infty} g_{T}\left(\underline{k}, \underline{l}_{1}, \ldots, \underline{l}_{r}\right)= \begin{cases}1, & \text { if } \quad\left(\underline{k}, \underline{l}_{1}, \ldots, \underline{l}_{r}\right)=(\underline{0}, \underline{0}, \ldots, \underline{0}) \\ 0, & \text { if } \quad\left(\underline{k}, \underline{l}_{1}, \ldots, \underline{l}_{r}\right) \neq(\underline{0}, \underline{0}, \ldots, \underline{0}) .\end{cases}
$$

This proves the lemma. 
Let $\sigma_{1}>\frac{1}{2}$ be a fixed number, and

$$
\begin{aligned}
& v(m, n)=\exp \left\{-\left(\frac{m}{n}\right)^{\sigma_{1}}\right\}, \quad m, n \in \mathbb{N}, \\
& v_{j}(m, n)=v_{j}\left(m, n, \alpha_{j}\right)=\exp \left\{-\left(\frac{m+\alpha_{j}}{n+\alpha_{j}}\right)^{\sigma_{1}}\right\}, \quad m \in \mathbb{N}_{0}, n \in \mathbb{N} .
\end{aligned}
$$

Define the series

$$
\begin{aligned}
& L_{n}\left(s, \chi_{j}\right)=\sum_{m=1}^{\infty} \frac{\chi_{j}(m) v(m, n)}{m^{s}}, \quad j=1, \ldots, l, \\
& \zeta_{n}\left(s, \alpha_{j}\right)=\sum_{m=0}^{\infty} \frac{v_{j}(m, n)}{\left(m+\alpha_{j}\right)^{s}}, j=1, \ldots, r, \\
& L_{n}\left(s, \hat{\omega}, \chi_{j}\right)=\sum_{m=1}^{\infty} \frac{\chi_{j}(m) \hat{\omega}(m) v(m, n)}{m^{s}}, \quad j=1, \ldots, l, \\
& \zeta_{n}\left(s, \alpha_{j}, \omega_{j}\right)=\sum_{m=0}^{\infty} \frac{\omega_{j}(m) v_{j}(m, n)}{\left(m+\alpha_{j}\right)^{s}}, \quad j=1, \ldots, r .
\end{aligned}
$$

It follows by a standard way that the series for $L_{n}\left(s, \chi_{j}\right), \zeta_{n}\left(s, \alpha_{j}\right)$ and $L_{n}(s, \hat{\omega}$, $\left.\chi_{j}\right), \zeta_{n}\left(s, \alpha_{j}, \omega_{j}\right)$ converge absolutely for $\sigma_{1}>\frac{1}{2}$. Define the probability measures

$$
\begin{aligned}
P_{T, n}(A)= & \frac{1}{T} \operatorname{meas}\left\{\tau \in[0, T]:\left(L_{n}\left(s+i \tau, \chi_{1}\right), \ldots, L_{n}\left(s+i \tau, \chi_{l}\right),\right.\right. \\
& \left.\left.\zeta_{n}\left(s+i \tau, \alpha_{1}\right), \ldots, \zeta_{n}\left(s+i \tau, \alpha_{r}\right)\right) \in A\right\}, \quad A \in \mathcal{B}\left(H^{l+r}(D)\right), \\
\hat{P}_{T, n}(A)= & \frac{1}{T} \operatorname{meas}\left\{\tau \in[0, T]:\left(L_{n}\left(s+i \tau, \hat{\omega}, \chi_{1}\right), \ldots, L_{n}\left(s+i \tau, \hat{\omega}, \chi_{l}\right),\right.\right. \\
& \left.\left.\zeta_{n}\left(s+i \tau, \alpha_{1}, \omega_{1}\right), \ldots, \zeta_{n}\left(s+i \tau, \alpha_{r}, \omega_{r}\right)\right) \in A\right\}, \quad A \in \mathcal{B}\left(H^{l+r}(D)\right) .
\end{aligned}
$$

Lemma 1, the absolute convergence of the above series and a property of the weak convergence of probability measures related by continuous mappings imply the following lemma.

Lemma 2. Suppose that the set $L\left(\mathcal{P}, \alpha_{1}, \ldots, \alpha_{r}\right)$ is linearly independent over $\mathbb{Q}$. Then, on $\left(H^{l+r}(D), \mathcal{B}\left(H^{l+r}(D)\right)\right)$, there exists a probability measure $P_{n}$ such that both the measures $P_{T, n}$ and $\hat{P}_{T, n}$ converges weakly to $P_{n}$ as $T \rightarrow \infty$.

The next step of the proof of Proposition 1 consists of the approximation of the functions $L\left(s, \chi_{j}\right)$ and $\zeta\left(s, \alpha_{j}\right)$ by $L_{n}\left(s, \chi_{j}\right)$ and $\zeta_{n}\left(s, \alpha_{j}\right)$, respectively. Let $\rho$ be the metric on $H(D)$ inducing the topology of uniform convergence on compacta, see, for example, [8]. Define the metric on $H^{l+r}(D)$ by

$$
\underline{\rho}\left(\underline{g}_{1}, \underline{g}_{2}\right)=\max _{1 \leq j \leq l+r} \rho\left(g_{1 j}, g_{2 j}\right)
$$

where $\underline{g}_{j}=\left(g_{j, 1}, \ldots, g_{j, l+r}\right) \in H^{l+r}(D), j=1,2$. Using the known onedimensional results of [7] and [15] for periodic zeta-functions, we obtain approximations in the space $H^{l+r}(D)$. 
Lemma 3. We have

$$
\begin{aligned}
\lim _{n \rightarrow \infty} & \limsup _{T \rightarrow \infty} \frac{1}{T} \int_{0}^{T} \underline{\rho}\left(\left(L\left(s+i \tau, \chi_{1}\right), \ldots, L\left(s+i \tau, \chi_{l}\right), \zeta\left(s+i \tau, \alpha_{1}\right), \ldots,\right.\right. \\
& \left.\quad\left(s+i \tau, \alpha_{r}\right)\right),\left(L_{n}\left(s+i \tau, \chi_{1}\right), \ldots, L_{n}\left(s+i \tau, \chi_{l}\right), \zeta_{n}\left(s+i \tau, \alpha_{1}\right), \ldots,\right. \\
& \left.\left.\zeta_{n}\left(s+i \tau, \alpha_{r}\right)\right)\right) \mathrm{d} \tau=0 .
\end{aligned}
$$

In the case of $\zeta\left(s, \alpha_{j}, \omega_{j}\right)$, a one-dimensional approximation is obtained for transcendental $\alpha_{j}$, however, the transcendence is used only for a linear independence of the set $\left\{\log \left(m+\alpha_{j}\right): m \in \mathbb{N}_{0}\right\}$. Therefore, we have the following analogue of Lemma 3 .

Lemma 4. Suppose that the set $L\left(\mathcal{P}, \alpha_{1}, \ldots, \alpha_{r}\right)$ is linearly independent over $\mathbb{Q}$. Then, for almost all $\underline{\omega} \in \underline{\Omega}$,

$$
\begin{aligned}
\lim _{n \rightarrow \infty} & \limsup _{T \rightarrow \infty} \frac{1}{T} \int_{0}^{T} \underline{\rho}\left(\left(L\left(s+i \tau, \hat{\omega}, \chi_{1}\right), \ldots, L\left(s+i \tau, \hat{\omega}, \chi_{l}\right), \zeta\left(s+i \tau, \alpha_{1}, \omega_{1}\right)\right.\right. \\
& \left.\ldots, \zeta\left(s+i \tau, \alpha_{r}, \omega_{r}\right)\right),\left(L_{n}\left(s+i \tau, \hat{\omega}, \chi_{1}\right), \ldots, L_{n}\left(s+i \tau, \hat{\omega}, \chi_{l}\right)\right. \\
& \left.\left.\zeta_{n}\left(s+i \tau, \alpha_{1}, \omega_{1}\right), \ldots, \zeta_{n}\left(s+i \tau, \alpha_{r}, \omega_{r}\right)\right)\right) \mathrm{d} \tau=0
\end{aligned}
$$

Define one more probability measure

$$
\begin{aligned}
\hat{P}_{T}(A)= & \frac{1}{T} \operatorname{meas}\left\{\tau \in[0, T]:\left(L\left(s+i \tau, \hat{\omega}, \chi_{1}\right), \ldots, L\left(s+i \tau, \hat{\omega}, \chi_{l}\right),\right.\right. \\
& \left.\left.\zeta\left(s+i \tau, \alpha_{1}, \omega_{1}\right), \ldots, \zeta\left(s+i \tau, \alpha_{r}, \omega_{r}\right)\right) \in A\right\}, \quad A \in \mathcal{B}\left(H^{l+r}(D)\right) .
\end{aligned}
$$

Lemma 5. Suppose that the set $L\left(\mathcal{P}, \alpha_{1}, \ldots, \alpha_{r}\right)$ is linearly independent over $\mathbb{Q}$. Then, on $\left(H^{l+r}(D), \mathcal{B}\left(H^{l+r}(D)\right)\right)$, there exists a probability measure $P$ such that both the measures $P_{T}$ and $\hat{P}_{T}$ converges weakly to $P$ as $T \rightarrow \infty$.

The lemma is a consequence of Lemmas $2-4$. Its proof runs similarly to that of Theorem 6 from [10].

For the proof of Proposition 1, it is sufficient to show that the measure $P$ in Lemma 5 coincides with $P_{\Xi}$. For this, again the linear independence of the set $L\left(\mathcal{P}, \alpha_{1}, \ldots, \alpha_{r}\right)$ is applied.

Let $\underline{a}_{\tau}=\left\{\left(p^{-i \tau}: p \in \mathcal{P}\right),\left(\left(m+\alpha_{1}\right)^{-i \tau}: m \in \mathbb{N}_{0}\right), \ldots,\left(\left(m+\alpha_{r}\right)^{-i \tau}: m \in\right.\right.$ $\left.\left.\mathbb{N}_{0}\right)\right\}$ for $\tau \in \mathbb{R}$. Define a family $\left\{\Phi_{\tau}: \tau \in \mathbb{R}\right\}$ of measurable measure preserving transformations on $\underline{\Omega}$ by the formula $\Phi_{\tau}(\underline{\omega})=\underline{a}_{\tau} \underline{\omega}, \underline{\omega} \in \underline{\Omega}$. This family is a one-parameter group. A set $A \in \mathcal{B}(\underline{\Omega})$ is called invariant with respect to the group $\left\{\Phi_{\tau}: \tau \in \mathbb{R}\right\}$ if, for each $\tau \in \mathbb{R}$, the sets $A$ and $A_{\tau}=\Phi_{\tau}(A)$ may differ one from another only by $m_{H}$-measure zero. All invariant sets form a $\sigma$-subfield of $\mathcal{B}(\underline{\Omega})$. The group $\left\{\Phi_{\tau}: \tau \in \mathbb{R}\right\}$ is ergodic, if the latter $\sigma$-subfield consists only of the sets of $m_{H}$-measure zero or one.

Lemma 6. Suppose that the set $L\left(\mathcal{P}, \alpha_{1}, \ldots, \alpha_{r}\right)$ is linearly independent over $\mathbb{Q}$. Then the group $\left\{\Phi_{\tau}: \tau \in \mathbb{R}\right\}$ is ergodic. 
Proof. A method of Fourier transform is used, and the arguments are similar to those of Lemma 7 from [10], where the case of the algebraically independent numbers $\alpha_{1}, \ldots, \alpha_{r}$ was considered.

Proof of Proposition 1 . On the probability space $\left(\underline{\Omega}, \mathbb{B}(\underline{\Omega}), m_{H}\right)$, define a random variable $\xi$ by the formula

$$
\xi(\underline{\omega})= \begin{cases}1, & \text { if } \Xi(s, \underline{\omega}) \in A, \\ 0, & \text { if } \Xi(s, \underline{\omega}) \notin A,\end{cases}
$$

where $A$ is a fixed continuity set of the measure $P$ in Lemma 5 . Thus

$$
\lim _{T \rightarrow \infty} P_{T}(A)=P(A) .
$$

Lemma 6 implies the ergodicity of the random process $\xi\left(\Phi_{\tau}(\underline{\omega})\right)$. Therefore, by the Birkhoff-Khintchine theorem,

$$
\lim _{T \rightarrow \infty} \frac{1}{T} \int_{0}^{T} \xi\left(\Phi_{\tau}(\underline{\omega})\right) \mathrm{d} \tau=\mathbb{E} \xi
$$

where $\mathbb{E} \xi$ is the expectation of $\xi$. On the other hand, the definitions of $\xi$ and $\Phi_{\tau}$ yield the equalities

$$
\mathbb{E} \xi=\int_{\underline{\Omega}} \xi \mathrm{d} m_{H}=P_{\Xi}(A), \quad \frac{1}{T} \int_{0}^{T} \xi\left(\Phi_{\tau}(\underline{\omega})\right) \mathrm{d} \tau=\hat{P}_{T}(A) .
$$

Combining the above four equalities, we find that $P(A)=P_{\Xi}(A)$. Since $A$ was arbitrary, this is true for all continuity sets of the measure $P$, hence, $P(A)=P_{\Xi}(A)$ for all $A \in \mathcal{B}\left(H^{l+r}(D)\right)$. The proposition is proved.

For the proof of universality theorems, we additionally need the support of the measure $P_{\Xi}$. Since the space $H^{l+r}(D)$ is separable, the support of $P_{\Xi}$ is a minimal closed set $S_{\Xi} \in \mathcal{B}\left(H^{l+r}(D)\right)$ such that $P_{\Xi}\left(S_{\Xi}\right)=1$.

Proposition 2. Suppose that $\chi_{1}, \ldots, \chi_{l}$ are pairwise non-equivalent Dirichlet characters, and that the set $L\left(\mathcal{P}, \alpha_{1}, \ldots, \alpha_{r}\right)$ is linearly independent over $\mathbb{Q}$. Then the support of the measure $P_{\Xi}$ is the set $S^{l} \times H^{r}(D)$.

Proof. Since the characters $\chi_{1}, \ldots, \chi_{l}$ are pairwise non-equivalent, the support of the random element

$$
\underline{L}\left(s, \hat{\omega}, \chi_{1}, \ldots, \chi_{l}\right)=\left(L\left(s, \hat{\omega}, \chi_{1}\right), \ldots, L\left(s, \hat{\omega}, \chi_{l}\right)\right)
$$

is the set $S^{l}[11]$. The linear independence of the set $L\left(\mathcal{P}, \alpha_{1}, \ldots, \alpha_{r}\right)$ implies that of the set $L\left(\alpha_{1}, \ldots, \alpha_{r}\right)$. Therefore, by [9], the support of the random element $\underline{\zeta}\left(s, \alpha_{1}, \ldots, \alpha_{r}, \omega_{1}, \ldots, \omega_{r}\right)=\left(\zeta\left(s, \alpha_{1}, \omega_{1}\right), \ldots, \zeta\left(s, \alpha_{r}, \omega_{r}\right)\right)$ is $H^{r}(D)$. Since the spaces $H^{l}(D)$ and $H^{r}(D)$ are separable, we have [3] that

$$
\mathcal{B}\left(H^{l+r}(D)\right)=\mathcal{B}\left(H^{l}(D)\right) \times \mathcal{B}\left(H^{r}(D)\right) .
$$


Let $m_{H}^{r}$ be the Haar measure on $\left(\prod_{j=1}^{r} \Omega_{j}, \mathcal{B}\left(\prod_{j=1}^{r} \Omega_{j}\right)\right)$. Then $m_{H}$ is the product of the measures $\hat{m}_{H}$ and $m_{H}^{r}$. Let $A_{1} \in \mathcal{B}\left(H^{l}(D)\right)$ and $A_{2} \in \mathcal{B}\left(H^{r}(D)\right)$. Then, in view of (2.2), it suffices to consider $P_{\Xi}(A)$ with $A=A_{1} \times A_{2}$. Thus, by the above remark,

$$
\begin{gathered}
P_{\Xi}(A)=m_{H}(\underline{\omega} \in \underline{\Omega}: \Xi(s, \underline{\omega}) \in A)=\hat{m}_{H}\left(\hat{\omega} \in \hat{\Omega}: \underline{L}\left(s, \hat{\omega}, \chi_{1}, \ldots, \chi_{l}\right) \in A_{1}\right) \\
\times m_{H}\left(\left(\omega_{1}, \ldots, \omega_{r}\right) \in \prod_{j=1}^{r} \Omega_{j}: \underline{\zeta}\left(s, \alpha_{1}, \ldots, \alpha_{r}, \omega_{1}, \ldots, \omega_{r}\right) \in A_{2}\right) .
\end{gathered}
$$

This, together with supports of the random elements $\underline{L}\left(s, \hat{\omega}, \chi_{1}, \ldots, \chi_{l}\right)$ and $\underline{\zeta}\left(s, \alpha_{1}, \ldots, \alpha_{r}, \omega_{1}, \ldots, \omega_{r}\right)$ proves the proposition.

Now we are ready to prove a joint universality theorem for a collection $L\left(s, \chi_{1}\right), \ldots, L\left(s, \chi_{l}\right), \zeta\left(s, \alpha_{1}\right), \ldots, \zeta\left(s, \alpha_{r}\right)$.

Theorem 15. Suppose that $\chi_{1}, \ldots, \chi_{l}$ are pairwise non-equivalent Dirichlet characters, and that the set $L\left(\mathcal{P}, \alpha_{1}, \ldots, \alpha_{r}\right)$ is linearly independent over $\mathbb{Q}$. For $j=1, \ldots, l$, let $K_{j} \subset D$ be a compact subset with connected complement, and let $f_{j}(s)$ be a continuous non-vanishing function on $K_{j}$ which is analytic in the interior of $K_{j}$. For $j=1, \ldots, r$, let $\hat{K}_{j} \subset D$ be a compact subset with connected complement, and let $\hat{f}_{j}(s)$ be a continuous function on $\hat{K}_{j}$ which is analytic in the interior of $\hat{K}_{j}$. Then, for every $\varepsilon>0$,

$$
\begin{gathered}
\liminf _{T \rightarrow \infty} \frac{1}{T} \operatorname{meas}\left\{\tau \in[0, T]: \sup _{1 \leq j \leq l} \sup _{s \in K_{j}}\left|L\left(s+i \tau, \chi_{j}\right)-f_{j}(s)\right|<\varepsilon,\right. \\
\left.\sup _{1 \leq j \leq r} \sup _{s \in \hat{K}_{j}}\left|\zeta\left(s+i \tau, \alpha_{j}\right)-\hat{f}_{j}(s)\right|<\varepsilon\right\}>0 .
\end{gathered}
$$

Proof. By the Mergelyan theorem [16], see also [24], there exist polynomials $p_{j}(s), j=1, \ldots, l$, and $q_{j}(s), j=1, \ldots, r$, such that

$$
\begin{aligned}
& q_{j}(s), j=1, \ldots, r, \text { such that } \\
& \sup _{1 \leq j \leq l} \sup _{s \in K_{j}}\left|f_{j}(s)-e^{p_{j}(s)}\right|<\varepsilon / 2, \\
& \sup _{1 \leq j \leq r} \sup _{s \in \hat{K}_{j}}\left|\hat{f}_{j}(s)-q_{j}(s)\right|<\varepsilon / 2 .
\end{aligned}
$$

Define the set

$$
\begin{aligned}
G=\left\{\left(g_{1}, \ldots, g_{l}, \hat{g}_{1}, \ldots, \hat{g}_{r}\right) \in H(D)\right. & : \sup _{1 \leq j \leq l} \sup _{s \in K_{j}}\left|g_{j}(s)-e^{p_{j}(s)}\right|<\varepsilon / 2, \\
& \left.\sup _{1 \leq j \leq r} \sup _{s \in \hat{K}_{j}}\left|\hat{g}_{j}(s)-q_{j}(s)\right|<\varepsilon / 2\right\} .
\end{aligned}
$$

Then, in view of Proposition 2, $G$ is an open neighbourhood of the element $\left(e^{p_{1}}, \ldots, e^{p_{l}}, q_{1}, \ldots, q_{r}\right)$ of the support of the measure $P_{\Xi}$. Thus, $P_{\Xi}(G)>0$. Therefore, by Proposition 1,

$$
\begin{array}{r}
\liminf _{T \rightarrow \infty} \frac{1}{T} \text { meas }\left\{\tau \in[0, T]:\left(L\left(s+i \tau, \chi_{1}\right), \ldots, L\left(s+i \tau, \chi_{l}\right), \zeta\left(s+i \tau, \alpha_{1}\right),\right.\right. \\
\left.\left.\ldots, \zeta\left(s+i \tau, \alpha_{r}\right)\right) \in G\right\} \geq P_{\Xi}(G)>0 .
\end{array}
$$

From this, the definition of $G$ and equalities (2.3) and (2.4), the theorem follows. 
Proposition 3. Suppose that the set $L\left(\mathcal{P}, \alpha_{1}, \ldots, \alpha_{r}\right)$ is linearly independent over $\mathbb{Q}$, and that $F \in U_{l, r}$. Then the probability measure

$$
\begin{aligned}
P_{T, F}(A) \stackrel{\text { def }}{=} & \frac{1}{T} \operatorname{meas}\left\{\tau \in[0, T]: F\left(L\left(s+i \tau, \chi_{1}\right), \ldots, L\left(s+i \tau, \chi_{l}\right),\right.\right. \\
& \left.\left.\zeta\left(s+i \tau, \alpha_{1}\right), \ldots, \zeta\left(s+i \tau, \alpha_{r}\right)\right) \in A\right\}, \quad A \in \mathcal{B}(H(D)),
\end{aligned}
$$

converges weakly to $P_{\Xi} F^{-1}$ as $T \rightarrow \infty$.

Proof. The proposition is a consequence of Proposition 1, of the continuity of $F$ and Theorem 5.1 from [3].

Proposition 4. Suppose that $\chi_{1}, \ldots, \chi_{l}$ are pairwise non-equivalent Dirichlet characters, the set $L\left(\mathcal{P}, \alpha_{1}, \ldots, \alpha_{r}\right)$ is linearly independent over $\mathbb{Q}$, and that $F \in U_{l, r}$. Then the support of the measure $P_{\Xi} F^{-1}$ is the whole of $H(D)$.

Proof. Let $g$ be an arbitrary element of $H(D)$, and $G$ be its open neighbourhood. From the continuity of $F$, we have that the $F^{-1} G$ is open, too.

It is well known that the approximation in the space $H(D)$ reduces to an approximation on compact subsets with connected complement, see, for example, [13]. Therefore, by the Mergelyan theorem, there exists a polynomial $p=p(s)$ such that $p \in G$. Since, by the hypothesis of the proposition, the set $\left(F^{-1}\{p\}\right) \cap\left(S^{l} \times H^{r}(D)\right)$ is non-empty, we have that the set $\left(F^{-1} G\right) \cap$ $\left(S^{l} \times H^{r}(D)\right)$ also is non-empty. This means that the set $F^{-1} G$ is an open neighbourhood of the element $g \in S^{l} \times H^{r}(D)$. In view of Proposition 2, the set $S^{l} \times H^{r}(D)$ is the support of the measure $P_{\Xi}$, thus $P_{\Xi}\left(F^{-1} G\right)>0$. Hence,

$$
P_{\Xi} F^{-1}(G)=P_{\Xi}\left(F^{-1} G\right)>0 .
$$

Since $g$ and $G$ are arbitrary, this proves the proposition.

Theorem 16. Suppose that $\chi_{1}, \ldots, \chi_{l}$ are pairwise non-equivalent Dirichlet characters, and that the set $L\left(\mathcal{P}, \alpha_{1}, \ldots, \alpha_{r}\right)$ is linearly independent over $\mathbb{Q}$, and that $F \in U_{l, r}$. Let $K \subset D$ be a compact set with connected complement, and $f(s)$ be a continuous function on $K$ which is analytic in the interior of $K$. Then, for every $\varepsilon>0$,

$$
\begin{array}{r}
\liminf _{T \rightarrow \infty} \frac{1}{T} \operatorname{meas}\left\{\tau \in[0, T]: \sup _{1 \leq j \leq l} \sup _{s \in K_{j}} \mid F\left(L\left(s+i \tau, \chi_{1}\right), \ldots, L\left(s+i \tau, \chi_{l}\right)\right.\right. \\
\left.\left.\zeta\left(s+i \tau, \alpha_{1}\right), \ldots, \zeta\left(s+i \tau, \alpha_{r}\right)\right)-f(s) \mid<\varepsilon\right\}>0 .
\end{array}
$$

Proof. We repeat the arguments similar to those of the proof of Theorem 15. By the Mergelyan theorem, there exists a polynomial $p(s)$ such that

$$
\sup _{s \in K}|f(s)-p(s)|<\frac{\varepsilon}{2} \text {. }
$$

Define the set

$$
G=\left\{g \in H(D): \sup _{s \in K}|g(s)-p(s)|<\frac{\varepsilon}{2}\right\}
$$


Then, by Proposition 4, the set $G$ is an open neighbourhood of the element $p(s)$ of the support of $P_{\Xi} F^{-1}$. Therefore, Proposition 3 implies the inequality

$$
\begin{array}{r}
\liminf _{T \rightarrow \infty} \frac{1}{T} \operatorname{meas}\left\{\tau \in[0, T]: F\left(L\left(s+i \tau, \chi_{1}\right), \ldots, L\left(s+i \tau, \chi_{l}\right),\right.\right. \\
\left.\left.\zeta\left(s+i \tau, \alpha_{1}\right), \ldots, \zeta\left(s+i \tau, \alpha_{r}\right)\right) \in G\right\} \geq P_{\Xi} F^{-1}(G)>0 .
\end{array}
$$

Combining this with (2.5) and the definition of $G$ completes the proof.

\section{Proof of the main theorems}

Proofs of all theorems on zeros are based on the corresponding universality theorems as well as on the classical Rouché theorem.

Proof of Theorem 2. We take $f(s)=s-\sigma_{0}$ in Theorem 10. Then, by Theorem 10 , for every $\varepsilon>0$, the set of $\tau \in \mathbb{R}$ satisfying the inequality

$$
\sup _{\left|s-\sigma_{0}\right| \leq \rho_{0}}\left|F\left(L\left(s+i \tau, \chi_{1}\right), \ldots, L\left(s+i \tau, \chi_{n}\right)\right)-\left(s-\sigma_{0}\right)\right|<\varepsilon
$$

has a positive lower density. Now we take $\varepsilon$ such that

$$
0<\varepsilon<\frac{1}{10} \inf _{\left|s-\sigma_{0}\right|=\rho_{0}}\left|s-\rho_{0}\right|=\frac{\rho_{0}}{10} .
$$

Thus, the functions $s-\sigma_{0}$ and $F\left(L\left(s+i \tau, \chi_{1}\right), \ldots, L\left(s+i \tau, \chi_{n}\right)\right)-\left(s-\sigma_{0}\right)$ on the disc $\left|s-\sigma_{0}\right| \leq \rho_{0}$ satisfy the hypotheses of the Rouché theorem. This proves the theorem.

Proof of Theorem 3. We use Theorem 11 and repeat the proof of Theorem 2.

Proof of Theorem 4. We use the notation

$$
\sigma_{0}=\frac{\sigma_{1}+\sigma_{2}}{2}, \quad \rho_{0}=\frac{\sigma_{2}-\sigma_{1}}{2}, \quad C=\sum_{j=1}^{n}\left|c_{j}\right| .
$$

Without loss of generality, we may suppose that $c_{1} \neq 0$. Let

$$
f_{1}(s)=c_{1}^{-1}\left(s-\sigma_{0}\right), \quad f_{2}(s)=\cdots=f_{n}(s)=\varepsilon>0,
$$

where the number $\varepsilon$ satisfies inequality

$$
C \varepsilon<\frac{1}{20} \min _{\left|s-\sigma_{0}\right|=\rho_{0}}\left|s-\sigma_{0}\right|=\frac{\rho_{0}}{20} .
$$

Suppose that reals $\tau$ satisfy the inequality

$$
\sup _{1 \leq j \leq n} \sup _{1 s-\sigma_{0} \mid \leq \rho_{0}}\left|\zeta\left(s+i \tau, \alpha_{j}\right)-f_{j}(s)\right|<\varepsilon .
$$


From this, we find that

$$
\sup _{\left|s-\sigma_{0}\right| \leq \rho_{0}}\left|\sum_{j=1}^{n} c_{j} \zeta\left(s+i \tau, \alpha_{j}\right)-\sum_{j=1}^{n} c_{j} f_{j}(s)\right|<C \varepsilon .
$$

Therefore, by the definition of $f_{1}(s), \ldots, f_{n}(s)$,

$$
\sup _{\left|s-\sigma_{0}\right|=\rho_{0}}\left|\sum_{j=1}^{n} c_{j} \zeta\left(s+i \tau, \alpha_{j}\right)-\left(s-\sigma_{0}\right)\right|<2 C \varepsilon
$$

and the proof finishes by application of Theorem 12 and the Rouché theorem.

Proof of Theorem 5. We use Theorem 13 and the Rouché theorem, and argue similarly to the proof of Theorem 2 .

Proof of Theorem 6. The proof coincides with that of Theorem 5. In place of Theorem 13, we apply Theorem 14.

Proof of Theorem 7 . Without loss of generality, we may assume that $\hat{c}_{1} \neq 0$. We take in Theorem 15

$$
f_{1}(s)=\cdots=f_{l}(s)=\varepsilon, \quad \hat{f}_{1}(s)=\hat{c}_{1}^{-1}\left(s-\sigma_{0}\right), \quad \hat{f}_{2}(s)=\cdots=\hat{f}_{r}(s)=\varepsilon,
$$

where the positive number $\varepsilon$ satisfies the inequality

$$
\left(C_{1}+C_{2}\right) \varepsilon<\frac{1}{20} \min _{\left|s-\sigma_{0}\right|=\rho_{0}}\left|s-\rho_{0}\right|=\frac{\rho_{0}}{20}
$$

and

$$
C_{1}=\sum_{j=1}^{l}\left|c_{j}\right|, \quad C_{2}=\sum_{j=1}^{r}\left|\hat{c}_{j}\right| .
$$

Let $\tau$ satisfy the inequalities

$$
\sup _{1 \leq j \leq l} \sup _{\left|s-\sigma_{0}\right| \leq \rho_{0}}\left|L\left(s+i \tau, \chi_{j}\right)-f_{j}(s)\right|<\varepsilon
$$

and

$$
\sup _{1 \leq j \leq r} \sup _{\left|s-\sigma_{0}\right| \leq \rho_{0}}\left|\zeta\left(s+i \tau, \alpha_{j}\right)-\hat{f}_{j}(s)\right|<\varepsilon .
$$

Then, for these $\tau$, we find that

$$
\begin{array}{r}
\sup _{\left|s-\sigma_{0}\right| \leq \rho_{0}} \mid \sum_{j=1}^{l} c_{j} L\left(s+i \tau, \chi_{j}\right)+\sum_{j=1}^{r} \hat{c}_{j} \zeta\left(s+i \tau, \alpha_{j}\right)-\sum_{j=1}^{l} c_{j} f_{j}(s) \\
-\sum_{j=1}^{r} \hat{c}_{j} \hat{f}_{j}(s) \mid<\left(C_{1}+C_{2}\right) \varepsilon .
\end{array}
$$


Moreover,

$$
\sup _{\left|s-\sigma_{0}\right| \leq \rho_{0}}\left|\sum_{j=1}^{l} c_{j} f_{j}(s)+\sum_{j=1}^{r} \hat{c}_{j} \hat{f}_{j}(s)-\left(s-\sigma_{0}\right)\right|<\left(C_{1}+C_{2}\right) \varepsilon .
$$

Two latter inequalities show that

$$
\sup _{\left|s-\sigma_{0}\right|=\rho_{0}}\left|\sum_{j=1}^{l} c_{j} L\left(s+i \tau, \chi_{j}\right)+\sum_{j=1}^{r} \hat{c}_{j} \zeta\left(s+i \tau, \alpha_{j}\right)-\left(s-\sigma_{0}\right)\right|<2\left(C_{1}+C_{2}\right) \varepsilon .
$$

From this and the definition of $\varepsilon$, it follows that the functions

$$
\sum_{j=1}^{l} c_{j} L\left(s+i \tau, \chi_{j}\right)+\sum_{j=1}^{r} \hat{c}_{j} \zeta\left(s+i \tau, \alpha_{j}\right)-\left(s-\sigma_{0}\right)
$$

and $s-\sigma_{0}$ in the disc $\left|s-\sigma_{0}\right| \leq \rho_{0}$ satisfy the hypotheses of the Rouché theorem. Hence, the theorem follows.

Proof of Theorem 8. We use Theorem 16 and repeat the proof, for example, of Theorem 2.

\section{References}

[1] B. Bagchi. The Statistical Behaviuor and Universality Properties of the Riemann Zeta-Function and Other Allied Dirichlet Series. PhD Thesis, Indian Statistical Institute, Calcutta, 1981.

[2] B. Bagchi. A joint universality theorem for Dirichlet L-functions. Math. Z., 181(3):319-334, 1982. https://doi.org/10.1007/BF01161980.

[3] P. Billingsley. Convergence of Probability Measures. Willey, New York, 1968.

[4] J.W.S. Cassels. Footnote to a note of Davenport and Heilbronn. J. London Math. Soc., 36:177-184, 1961. https://doi.org/10.1112/jlms/s1-36.1.177.

[5] H. Davenport and H. Heilbronn. On the zeros of certain Dirichlet series I. $J$. London Math. Soc., 11:181-185, 1936.

[6] S.M. Gonek. Analytic Properties of Zeta and L-Functions. PhD Thesis, University of Michigan, 1979.

[7] A. Javtokas and A. Laurinčikas. On the periodic Hurwitz zeta-function. HardyRamanujan J., 29:18-36, 2006.

[8] A. Laurinčikas. Limit Theorems for the Riemann Zeta-Function. Kluwer Academic Publishers, Dordrecht, Boston, London, 1996.

[9] A. Laurinčikas. The joint universality of Hurwitz zeta-functions. Šiauliai Math. Semin., 3 (11):169-187, 2008.

[10] A. Laurinčikas. Joint universality of zeta-functions with peri-

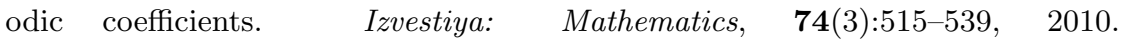
https://doi.org/10.1070/IM2010v074n03ABEH002497.

[11] A. Laurinčikas. On joint universality of Dirichlet L-functions. Chebyshevskii Sb., 12(1):124-139, 2011. 
[12] A. Laurinčikas. Joint universality of Hurwitz zeta-functions. Bull. Aust. Math. Soc., 86:232-243, 2012. https://doi.org/10.1017/S0004972712000342.

[13] A. Laurinčikas. Universality of composite functions. RIMS Kôkyôroku Bessatsu, B34:191-204, 2012.

[14] A. Laurinčikas and R. Garunkštis. The Lerch Zeta-Function. Kluwer Academic Publishers, Dordrecht, Boston, London, 2002.

[15] A. Laurinčikas and D. Šiaučiūnas. Remarks on the universality of periodic zetafunction. Math. Notes, 80(3-4):711-722, 2006.

[16] S.N. Mergelyan. Uniform approximations to functions of complex variable. Usp. Mat. Nauk., 7:31-122, 1952 (in Russian).

[17] A. Mikalajūnaitè. Joint universality of Dirichlet L-functions. Master thesis, Vilnius University, Faculty of Mathematics and Informatics, Vilnius, 2012 (in Lithuanian).

[18] T. Nakamura and $\mathrm{E}$. Pańkowski. On universality for linear combinations of $L$ functions. Monatsh. Math., 165:433-446, 2012. https://doi.org/10.1007/s00605011-0283-7.

[19] T. Nakamura and Ł. Pańkowski. On complex zeros off the critical line for non-monomial polynomial of zeta-functions. Math. Z., 284:23-39, 2016. https://doi.org/10.1007/s00209-016-1643-8.

[20] J. Steuding. Value-Distribution of L-Functions. Lect. Notes. Math. Vol. 1877, Springer-Verlag, Berlin, Heidelberg, 2007.

[21] S.M. Voronin. On the functional independence of Dirichlet L-functions. Acta Arith., 27:493-503, 1975 (in Russian).

[22] S.M. Voronin. Analytic Properties of Generating Function of Arithmetic Objects. Diss. doktor fiz.-matem. nauk, Moscow, 1977 (in Russian).

[23] S.M. Voronin. Collected Works. Mathematics. A.A. Karatsuba (Ed.), Izd. MGTU im. N. E. Baumana, Moscow, 2006 (in Russian).

[24] J.L. Walsh. Interpolation and Approximation by Rational Functions in the Complex Domain. Amer. Math. Soc. Colloq. Publ., Vol. 20, 1960. 